\title{
ENFERMEDAD ARTICULAR CRÓNICA COMO SECUELA DEL DENGUE
}

\section{CHRONIC JOINT DISEASE AS A SEQUEL TO DENGUE}

\author{
Barre-Lovaton Ángel Leonardo 1; Cornejo-Quiñonez Cesar Alonso 2; Solís-Olive Cecilia \\ del Carmen ${ }^{3}$; Rosero-Angulo Karen Eugenia ${ }^{4}$, Rosero-Montesuma Tamara Leonor ${ }^{5}$; \\ Salazar-Buenaño Julio Patricio ${ }^{6}$

\footnotetext{
${ }^{1}$ Pontifica Universidad Católica del Ecuador. Quito, Ecuador. Correo: albarre@puce.edu.ec.

${ }^{2}$ Pontifica Universidad Católica del Ecuador. Quito, Ecuador. Correo: cacornejo@puce.edu.ec.

${ }^{3}$ Pontifica Universidad Católica del Ecuador. Quito, Ecuador. Correo: cdsolis@puce.edu.ec.

${ }^{4}$ Pontifica Universidad Católica del Ecuador. Quito, Ecuador. Correo: kerosero@puce.edu.ec.

${ }^{5}$ Pontifica Universidad Católica del Ecuador. Quito, Ecuador. Correo: trosero@puce.edu.ec.

${ }^{6}$ Pontifica Universidad Católica del Ecuador. Quito, Ecuador. Correo: jpsalazar@puce.edu.ec.
}

\section{Resumen}

El objetivo de esta investigación se centró en determinar la relación entre dengue y enfermedad articular crónica, en pacientes atendidos durante el período 2018-2020 en el Centro de Salud Tipo C San Rafael de Esmeraldas. Se planteó un estudio de casos y controles. Para ello se buscó analizar a dos grupos de pacientes de ambos sexos en un rango de edad comprendido entre 18 a 65 años que fueron atendidos durante el período de tiempo entre enero del 2018 a diciembre del 2020 en el Centro de Salud Tipo C San Rafael. Los grupos estudiados fueron: Para los Casos se consideraron pacientes con diagnóstico de artropatía crónica como espondiloartritis (M469), artritis reumatoidea (M05), espondilitis anquilosante (M450), otras artritis (M130), otras artropatías especificas (M120), lupus eritematoso sistémico (M329), que acudieron durante enero del 2018 a diciembre del 2020 al Centro de Salud Tipo C San Rafael. Para el grupo control se consideraron pacientes sin artropatía inflamatoria crónica, reclutados en el Centro de Salud Tipo C San Rafael, entre mayo y junio 2021. En el estudio se incluyeron 147 individuos (entre 18 a 65 años) divididos en dos grupos, para el grupo control se contó con 112 individuos (65 mujeres y 47 hombres), mientras que el grupo caso se conformó con 35 individuos (27 mujeres y 8 hombres), siendo así que los resultados de la investigación arrojaron como resultado que el $77,1 \%$ (27 individuos) padece de un tipo de artritis no especificada (M139), así también el 17,1\% (6 individuos) mencionó tener lupus eritematoso sistémico, y el 5,7\% (2 individuos) restante dijo tener espondilitis anquilosante (M450). En base a los resultados obtenidos se evidenció que existió un riesgo 11,44 superior de padecer algún tipo enfermedad articular crónica en pacientes que reportaron dengue en algún momento de su vida. Así también se establece que el consumo de tabaco de los grupos caso control se estableció entre 1 y 10 cigarrillos diariamente, siendo este hábito un factor importante y estadísticamente significativo (Chi-cuadrado de 51.00) de considerar para la predisposición de las manifestaciones articulares crónicas evidenciadas.

Palabras clave: Dengue, enfermedad articular crónica, artritis reumatoide.

\begin{abstract}
The objective of this research focused on determining the relationship between dengue and chronic joint disease, in patients attended during the period 2018-2020 at the Type C Health Center San Rafael de Esmeraldas. A case-control study was proposed. For this purpose, we sought to analyze two groups of patients of both sexes in an age range from 18 to 65 years who were attended during the period from January 2018 to December 2020 at the Type C San Rafael Health Center. The groups studied were: for the Cases, patients with a diagnosis of chronic
\end{abstract}

Información del manuscrito:

Fecha de recepción: 17 de diciembre de 2021 .

Fecha de aceptación: 01 de febrero de 2022.

Fecha de publicación: 07 de febrero de 2022. 
arthropathy such as spondyloarthritis (M469), rheumatoid arthritis (M05), ankylosing spondylitis (M450), other arthritis (M130), other specific arthropathies (M120), systemic lupus erythematosus (M329), who attended during January 2018 to December 2020 at the Type C San Rafael Health Center were considered. For the control group, patients without chronic inflammatory arthropathy, recruited at the Type C San Rafael Health Center, between May and June 2021, were considered. The study included 147 individuals (between 18 and 65 years old) divided into two groups, for the control group there were 112 individuals ( 65 women and 47 men), while the case group consisted of 35 individuals ( 27 women and 8 men), Thus, the results of the investigation showed that $77.1 \%$ (27 individuals) suffer from an unspecified type of arthritis (M139), as well as $17.1 \%$ (6 individuals) mentioned having systemic lupus erythematosus, and the remaining 5.7\% (2 individuals) said they had ankylosing spondylitis (M450). Based on the results obtained, it was found that there was an 11.44 higher risk of suffering from some type of chronic joint disease in patients who reported dengue fever at some time in their lives. It was also established that the consumption of tobacco in the control case groups was between 1 and 10 cigarettes daily, being this habit an important and statistically significant factor (Chi-square of 51.00) to consider for the predisposition of the chronic joint manifestations evidenced.

Keywords: Dengue, chronic joint disease, rheumatoid arthritis.

\section{INTRODUCCIÓN}

El presente estudio pretende aclarar la asociación del dengue con la enfermedad articular crónica (EAC), en particular con artritis reumatoidea (AR). La información científica ha dejado claro que los factores que inciden en el desarrollo de la AR son ambiguos y dependen de cada región geográfica en particular, además hay evidencia que relaciona la infección con el virus del dengue (DG) con el desencadenamiento o la exacerbación de la AR.

El DG es una infección vírica transmitida por la picadura de las hembras infectadas de mosquitos del género Aedes, en especial del Aedes aegypti. Además, es una enfermedad que se puede presentar como un síndrome gripal, que afecta a lactantes, niños pequeños $y$ adultos, los síntomas son una fiebre elevada (40Cํ) acompañada de dos de los síntomas siguientes: cefalea intensa, dolor retroocular, dolores osteomioarticulares, náuseas, vómitos, agrandamiento de ganglios linfáticos o exantema (Organización Mundial de la Salud, 2017).

El Ministerio de Salud Pública del Ecuador menciona que el DG es una virosis endémica en el Ecuador al igual que sucede con otras infecciones virales y bacterianas, que representa un prioritario y creciente problema de salud pública en el contexto de las enfermedades transmitidas por vectores, y que 
además muestra un comportamiento endemo-epidémico desde su aparición a finales de 1988 (Ministerio de Salud Pública, s. f.)

De acuerdo con Lozano (2001), en la medicina una de las patologías que comúnmente se ven en las casas de salud son los trastornos articulares, muchos de estos de etiología desconocida, pero en los que el sistema inmune juega un papel fundamental en su desarrollo y evolución; menciona además que los mismos se caracterizan por causar discapacidad entre los individuos que la padecen.

De acuerdo con Mestanza (s. f.) en Ecuador la incidencia de enfermedades reumáticas alcanza el $0.9 \%$, siendo la más frecuente la artritis.

Por otra parte, la $A R$ es una enfermedad sistémica crónica de etiología desconocida que presenta manifestaciones

musculoesqueléticas graves, esta enfermedad se caracteriza por criterios como: rigidez matutina por alrededor de 1 hora, artritis en más de tres articulaciones, artritis simétrica, nódulos reumatoideos, factor reumatoideo positivo, alteraciones radiológicas compatibles con la enfermedad (Suárez y LLorente,2012).

Al establecer el DG como factor de riesgo para el desarrollo futuro de manifestaciones articulares, se espera crear una alerta al sistema de salud para hacer tamizaje de problemas articulares en pacientes que han sufrido dengue de forma oportuna, logrando así que su detección temprana contribuya a mejorar la calidad de vida del paciente.

\section{METODOLOGÍA}

Para el presente estudio se plantea un enfoque cuantitativo de tipo casos y controles.

\section{Materiales y métodos}

El estudio se llevó a cabo en el Centro de Salud Tipo C San Rafael, Distrito 08D01. Para ello se analizó a un total de 147 individuos, 92 mujeres y 55 hombres divididos en dos grupos, el primer grupo correspondiente a los "individuos considerados casos" y el segundo grupo a los "individuos considerados control", estos comprendían rangos de edades entre 18 a 65 años. Para el primer grupo correspondiente a los 
individuos "Casos" se consideró a pacientes que fueron atendidos durante el período de tiempo comprendido entre enero del 2018 a diciembre del 2020, mientras que para el segundo grupo se consideró a individuos entrevistados entre mayo y junio del 2021 que asistieron a esa casa de salud durante ese lapso.

Durante los meses de mayo a agosto del 2021 se clasificaron los pacientes en dos grupos:

GRUPO CASO: Se incluyeron en este grupo a 35 individuos que presentaron artropatía crónica, y que además aceptaron participar en el estudio, mismo que se encontraban en un rango de edad comprendida entre 18 a 65 años.

GRUPO CONTROL: Para este grupo se consideraron a 112 individuos que no tenían antecedente de artropatía crónica, además de que los mismos hayan aceptado participar en el estudio, así como encontrarse en un rango de edad comprendida entre 18 a 65 años.

Así mismo, como parte de la investigación se crearon criterios de excusión que permitieran un mejor control de los individuos participantes en el estudio, mismo que consintieron en:

GRUPO CASO: Osteoartritis, Personas menores de 18 y mayores a 65 años, imposibilidad de acceder a la historia clínica del paciente, imposibilidad para contactar al paciente si es requerido, no aceptar participar.

GRUPO CONTROL: No aceptar participar en el estudio.

\section{RESULTADOS}

Para poder realizar la interpretación de los datos obtenidos para el estudio fue necesario que tanto los datos de los 35 individuos del grupo caso (individuos que presentaron manifestaciones articulares crónicas), así como los datos de los 112 individuos participantes en el grupo control (individuos que no presentaron manifestaciones articulares crónicas) sean agrupados y ordenados en diferentes tablas (presentes en los anexos de este estudio); esto con la finalidad de analizar de formas más rápida la información que se recabó a través de las historias clínicas y entrevistas a participantes en el grupo caso, así 
como los datos obtenidos a través de entrevistas a los participantes en el grupo control.
De esta forma, luego de haber recabado los datos y ser ordenados para su interpretación en la tabla 1 los resultados en los grupos caso y control son los siguientes:

Tabla 1 Distribución de las variables analizadas en los grupos caso y control

\begin{tabular}{|c|c|c|c|c|c|}
\hline Variable & Categoría & $\begin{array}{c}\text { Grupo Con } \\
\text { Manifestacio } \\
\text { nes } \\
\text { Articulares } \\
\text { (Grupo Caso } \\
n=35 \text { ) }\end{array}$ & $\begin{array}{c}\text { Grupo Sin } \\
\text { Manifestacio } \\
\text { nes } \\
\text { Articulares } \\
\text { (Grupo } \\
\text { Control } \\
n=112 \text { ) }\end{array}$ & $\begin{array}{l}\text { Chi- } \\
\text { Cuadrado } \\
\text { Calculado }\end{array}$ & $\begin{array}{l}\text { Chi- } \\
\text { Cuadrado } \\
\text { Tabular }\end{array}$ \\
\hline Edad & $\begin{array}{l}18 \text { A } 29 \text { años } \\
30 \text { A } 41 \text { años } \\
42 \text { A } 53 \text { años } \\
54 \text { A } 65 \text { años }\end{array}$ & $\begin{array}{l}4 \\
6 \\
17 \\
8\end{array}$ & $\begin{array}{l}32 \\
45 \\
24 \\
11\end{array}$ & $\begin{array}{l}17.83341 \\
239\end{array}$ & $\begin{array}{l}7.814727 \\
903\end{array}$ \\
\hline Sexo & $\begin{array}{l}\text { Hombre } \\
\text { Mujer }\end{array}$ & $\begin{array}{l}8 \\
27 \\
\end{array}$ & $\begin{array}{l}47 \\
65\end{array}$ & $\begin{array}{l}4.158767 \\
326\end{array}$ & $\begin{array}{l}3.841458 \\
821\end{array}$ \\
\hline Etnia & $\begin{array}{l}\text { Mestizo/a } \\
\text { Afroecuatoriano/a } \\
\text { Blanco/a } \\
\text { Negro/a }\end{array}$ & $\begin{array}{l}31 \\
1 \\
1 \\
2\end{array}$ & $\begin{array}{l}74 \\
35 \\
3 \\
0\end{array}$ & $\begin{array}{l}17.07595 \\
409\end{array}$ & $\begin{array}{l}7.814727 \\
903\end{array}$ \\
\hline Educación & $\begin{array}{l}\text { Ninguna } \\
\text { Básico } \\
\text { Bachillerato } \\
\text { Superior }\end{array}$ & $\begin{array}{l}3 \\
4 \\
11 \\
17\end{array}$ & $\begin{array}{l}2 \\
21 \\
48 \\
41\end{array}$ & $\begin{array}{l}6.287490 \\
231\end{array}$ & $\begin{array}{l}7.814727 \\
903\end{array}$ \\
\hline Ocupación & $\begin{array}{l}\text { Desempleado } \\
\text { Trabajo dependiente } \\
\text { Trabajo independiente }\end{array}$ & $\begin{array}{l}5 \\
12 \\
18 \\
\end{array}$ & $\begin{array}{l}16 \\
44 \\
52\end{array}$ & $\begin{array}{l}0.315094 \\
017\end{array}$ & $\begin{array}{l}5.991464 \\
547\end{array}$ \\
\hline \begin{tabular}{|l|} 
Ingreso \\
Económico
\end{tabular} & $\begin{array}{l}\text { Alto } \\
\text { Medio } \\
\text { Bajo }\end{array}$ & $\begin{array}{l}0 \\
18 \\
17\end{array}$ & $\begin{array}{l}30 \\
71 \\
11\end{array}$ & $\begin{array}{l}94.01215 \\
975\end{array}$ & $\begin{array}{l}5.991464 \\
547\end{array}$ \\
\hline Tabaco & $\begin{array}{l}\text { No consume } \\
\text { Consume de } 1 \text { a } 10 \\
\text { unidades } \\
\text { Consume más de } 10 \\
\text { unidades }\end{array}$ & $\begin{array}{l}11 \\
21 \\
3\end{array}$ & $\begin{array}{l}100 \\
8 \\
4\end{array}$ & $\begin{array}{l}51.00117 \\
409\end{array}$ & $\begin{array}{l}5.991464 \\
547\end{array}$ \\
\hline Dengue & $\begin{array}{l}\text { Positivo } \\
\text { Negativo }\end{array}$ & $\begin{array}{l}28 \\
7\end{array}$ & $\begin{array}{l}29 \\
83\end{array}$ & $\begin{array}{l}32.89385 \\
744\end{array}$ & $\begin{array}{l}3.841458 \\
821\end{array}$ \\
\hline
\end{tabular}

Fuente: Autores 
La tabla 1 muestra una comparación entre los resultados de las variables sociodemográficas que se consideraron para el estudio en los grupos caso y control, así como los valores obtenidos en el cálculo de chi- cuadrado, así como el nivel de significancia de estas (Chicuadrado).

Se realizó una distribución por rango de edad siendo posible constatar en la Tabla 1 que en el grupo caso (individuos que presentaron manifestaciones articulares crónicas) en el rango comprendido entre los 18 a 29 años se encontraron 4 individuos $(11,4 \%)$, mientras que $6(17,1 \%)$ se encontraban en el rango de edad comprendido entre 30 a 41 años, 17 individuos $(48,6 \%)$ estaban en el rango de edad de 42 a 53 años, y finalmente los 8 individuos restantes $(22,9 \%)$ estaban en el rango de edad de 54-65 años. En contraparte, el $28,6 \%$ de individuos del grupo control estaban en el rango de edad comprendido entre 18 a 29 años, mientras que el $40,2 \%$ se encontraba en una edad comprendida entre $30 \mathrm{a}$ 41 años, así también en el rango de 42 a 53 años encontramos el 21,4\% de los individuos participantes y finalmente el $9,8 \%$ restante comprende el rango de 54 a 65 años.

Respecto a la distribución del sexo de los individuos en el grupo caso se evidenció que 8 son hombres y 27 son mujeres, es decir el $22,9 \%$ son hombres y el $77,1 \%$ son mujeres. Mientras que en el grupo control en lo que respecta a la distribución del sexo entre los participantes, se puede evidenciar que el $42,0 \%$ de participantes (47 individuos) son hombres y el 58,0\% (65 individuos) restante son mujeres.

En lo relacionado a las etnias con las que se identificaron los individuos del grupo caso se estableció que, se consideraron como mestizos 31 de ellos, lo que corresponde al $88,6 \%$ de datos recolectados; como afroecuatoriano se identificó solo 1 individuo (2,9\%), igualmente solo 1 de ellos $(2,9 \%)$ se reconoció como blanco, y finalmente 2 individuos $(5,7 \%)$ se autoidentificaron como negros. Por otra parte, en el grupo control se evidenció que el $66,1 \%$ de los individuos se identificaron como mestizos, mientras que el $31,3 \%$ y $2,7 \%$ restantes afirmaron que se identificaban como afroecuatorianos y blancos respectivamente, mientras 
que ninguno de ellos se consideró como negro.

En lo referente al nivel de educación de los individuos de grupo caso, se registró que el 8,6\% (3 individuos) no posee ningún nivel de educación, mientras que los que han cursado una educación básica corresponden al $11,4 \%$ (4 individuos), respecto a los individuos que han cursado el bachillerato son el $31,4 \% \quad(11$ individuos), y el $48,6 \%$ restante (17 individuos) han cursado un nivel superior. Así mismo, el nivel de educación de los participantes del grupo control estuvo predominado por educación superior ocupando el $36,6 \%$ del total, seguido por el bachillerato con un $42,9 \%$, mientras que la educación básica obtuvo el $18,8 \%$ y finalmente el $1,8 \%$ de los individuos de este grupo mencionó no poseer ningún tipo de educación.

En lo relacionado a la ocupación de los individuos del grupo caso se muestra que el 14,3\% (5 individuos) manifestaron encontrarse desempleados, el $34,3 \% \quad$ (12 individuos) mencionan tener un trabajo dependiente, mientras que el $51,4 \%$ (18 individuos) realizan un trabaja de forma independiente. En lo correspondiente a los individuos del grupo control se encontró que el $14,3 \%$ de ellos se encontraban desempleados, el 39,3\% tenían un trabajo dependiente mientras el $46,4 \%$ de ellos mencionaron realizar trabajos de tipo independiente.

Respecto a la distribución de ingresos económicos de los individuos del grupo caso, se muestra que el 51,4\% (18 individuos) manifiestan tener ingresos medios, y el $48,6 \%$ (17 individuos) restante mencionan que tienen ingresos bajos, mientras que ninguno de ellos considera tener ingresos altos. En relación con los ingresos económicos de los individuos del grupo control se estableció que el $26,8 \%$ de ellos poseen ingresos que consideran son altos, mientras que el 63,4\% manifestó que con su trabajo logran ingresos económicos que consideran como medios y el 9,8\% dijo que tiene ingresos bajos.

En la distribución del consumo de tabaco de los individuos del grupo caso los resultados mostraron que el $31,4 \%$ (11 individuos) manifiesta no consumir tabaco, el 60,0\% (21 individuos) afirma consumir de 1 a 10 unidades de tabaco, y el 8,6\% restante (3 individuos) dijo consumir más de 10 unidades de tabaco. Al 
analizar los mismos datos en los individuos del grupo control se encontró que el $89,3 \%$ de ellos afirmo no consumirlo, en contraparte el $7,1 \%$ de ellos dijo consumir al menos de 1 a 10 unidades y solo el $3,6 \%$ de ellos mencionó que consumen más de 10 unidades.

En el análisis comparativo entre los grupos caso y control en lo referente a los reportes de dengue en los individuos participantes en cada grupo la tabla 2 nos muestra que, el $80 \%$ de los individuos pertenecientes al grupo caso (individuos con manifestaciones articulares) presentaron al menos 1 episodio de dengue en el transcurso de su vida, y de igual forma en el grupo control (individuos sin manifestaciones articulares) se evidenció que el $25,9 \%$ de los participantes también afirmaron haber presentado dengue. Por otra parte, el $20 \%$ restante de participantes del grupo caso mencionó que no han presentado dengue, misma afirmación que comparte el $74,1 \%$ de los individuos del grupo control.

Tabla 2. Distribución del total de casos de dengue entre los grupos caso y control

\begin{tabular}{|c|c|c|c|c|}
\hline \multirow{2}{*}{\multicolumn{2}{|c|}{ Dengue }} & \multicolumn{2}{|c|}{ Manifestaciones_Articulares } & \multirow[b]{2}{*}{ Total } \\
\hline & & Presentes & Ausentes & \\
\hline \multirow[t]{2}{*}{ Positivo } & Recuento & 28 & 29 & 57 \\
\hline & $\begin{array}{l}\% \text { dentro de } \\
\text { Manifestaciones_Articulares }\end{array}$ & $80,0 \%$ & $25,9 \%$ & $38,8 \%$ \\
\hline \multirow[t]{2}{*}{ Negativo } & Recuento & 7 & 83 & 90 \\
\hline & $\begin{array}{l}\% \text { dentro de } \\
\text { Manifestaciones_Articulares }\end{array}$ & $20,0 \%$ & $74,1 \%$ & $61,2 \%$ \\
\hline \multirow[t]{2}{*}{ Total } & Recuento & 35 & 112 & 147 \\
\hline & $\begin{array}{l}\% \text { dentro de } \\
\text { Manifestaciones Articulares }\end{array}$ & $100,0 \%$ & $100,0 \%$ & $100,0 \%$ \\
\hline
\end{tabular}

Fuente: Autores.

La tabla 2 muestra una comparación de los porcentajes obtenido entre los reportes de casos positivos 0 negativos de dengue y la presencia o ausencia de las manifestaciones articulares entre los individuos que participaron en el estudio.
En la Tabla 3 se muestran resultados que manifiestan que en el grupo caso se evidenció que los individuos presentaban varias manifestaciones articulares entre las que se encontró que el $77,1 \%$ (27 individuos) de los que hicieron parte de este grupo padecen de un tipo de artritis no especificada (M139), así también el 
17,1\% (6 individuos) mencionó tener

tener espondilitis anquilosante lupus eritematoso sistémico (M329), (M450).

y el 5,7\% (2 individuos) restante dijo

Tabla 3 Manifestaciones articulares encontradas en el grupo caso

\begin{tabular}{|l|r|r|}
\hline Manifestaciones Articulares & Frecuencia & \multicolumn{1}{|c|}{ Porcentaje } \\
\hline Artritis, no especificada (M139) & 27 & 77,1 \\
Lupus eritematoso sistémico (M329) & 6 & 17,1 \\
Espondilitis anquilosante (M450) & 2 & 5,7 \\
\hline Total & 35 & 100,0 \\
\hline
\end{tabular}

Fuente: Autores.

La tabla 3 muestra una comparación de los porcentajes obtenido entre las manifestaciones articulares crónicas que se reportaron en el grupo caso.

Tabla 4 Estimación de riesgo de manifestación articular y dengue

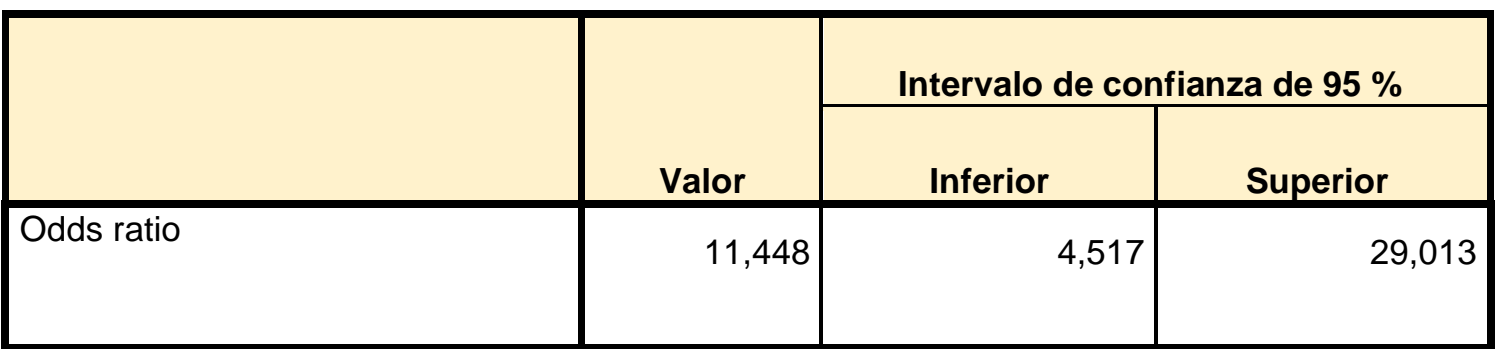

Fuente: Autores.

La tabla 4 muestra el riesgo de presentar manifestaciones articulares en pacientes que presentaron dengue.

Finalmente, de acuerdo con el cálculo del OR (Odds ratio) de la tabla 5 , es posible establecer que, los individuos que en algún momento han manifestado síntomas de haber contraído dengue muestran un riesgo 11.4 veces mayor de en el futuro desarrollar manifestaciones articulares crónicas a comparación de aquellos que mencionaron no haber tenido dengue.

\section{ANÁLISIS DE RESULTADOS}

A pesar de que en el estudio de Colman et al. (2017) en donde se habla del dengue y su impacto en pacientes con enfermedades autoinmunes, $y$ en donde se hace 
referencia respecto a la relación que guarda el dengue con la fisiopatología de las enfermedades autoinmunes, mencionando que este puede desencadenar o exacerbar las mismas. $Y$ además considerando que a nivel mundial es elevado el porcentaje de casos de dengue que se presentan cada año, en especial en países que cumplen con los requisitos necesarios para que el vector que lo transporta prolifere, actualmente aún no se han realizados suficientes estudios que permitan establecer una relación directa entre la infección por cualquier serotipo de dengue y el desarrollo de manifestaciones articulares crónicas.

Partiendo de estos antecedentes el estudio esperaba determinar si el dengue es un detonante para el desarrollo de manifestaciones articulares crónicas como artritis no identificadas, artritis reumatoidea, lupus eritematoso sistémico, entre otras patologías autoinmunes que cursan con manifestaciones articulares crónicas. Para esto se estableció la incidencia de dengue entre los individuos que desarrollaron patologías articulares crónicas respecto al sexo y al rango de edad en que se encontraban los mismo, puesto que en el estudio de Abumohor (2012) se menciona que la artritis reumatoidea (un tipo de manifestación articular crónica) es una afección que es más predominante en mujeres con una relación de dos mujeres por cada hombre, y que la misma es más frecuente de presentarse en individuos de 50 a 60 años.

Tabla 5 Distribución de las manifestaciones articulares por edad y sexo en grupo caso.

\begin{tabular}{|c|c|c|c|c|c|c|}
\hline & & & Manife & staciones Art & culares & \\
\hline & Eda & & $\begin{array}{c}\text { Artritis no } \\
\text { especificada } \\
\text { (M139) }\end{array}$ & \begin{tabular}{|c|} 
Lupus \\
eritematoso \\
sistémico \\
(M329)
\end{tabular} & $\begin{array}{c}\text { Espondilitis } \\
\text { anquilosante } \\
\text { (M450) }\end{array}$ & Total \\
\hline $18-29$ & Sexo & Hombre & 1 & 1 & & 2 \\
\hline & & Mujer & 0 & 2 & & 2 \\
\hline & Total & & 1 & 3 & & 4 \\
\hline $30-41$ & Sexo & Hombre & 1 & 0 & 0 & 1 \\
\hline & & Mujer & 1 & 3 & 1 & 5 \\
\hline & Total & & 2 & 3 & 1 & 6 \\
\hline $42-53$ & Sexo & $\begin{array}{l}\text { Hombre } \\
\text { Mujer }\end{array}$ & $\begin{array}{r}3 \\
14\end{array}$ & & & $\begin{array}{r}3 \\
14\end{array}$ \\
\hline & Total & & 17 & & & 17 \\
\hline $54-65$ & Sexo & Hombre & 2 & & 0 & 2 \\
\hline
\end{tabular}




\begin{tabular}{|lll|r|r|r|r|} 
& & Mujer & 5 & & 1 & 6 \\
& Total & & 7 & & 1 & 8 \\
\hline \multirow{2}{*}{ Total } & Sexo & Hombre & 7 & 1 & 0 & 8 \\
& Mujer & 20 & 5 & 2 & 27 \\
\cline { 2 - 6 } & Total & 27 & 6 & 2 & 35 \\
\hline
\end{tabular}

Fuente: Autores.

La tabla 5 muestra la frecuencia de casos presentados de dengue en relación con las manifestaciones articulares crónicas que se reportaron en el grupo caso.

Según la tabla 5 se muestra que en el estudio fue mayor la prevalencia (27 individuos) de artritis no especificada (M139) entre los individuos analizados, siendo más frecuente en mujeres (20 individuos) que se encontraban entre su cuarta y quinta década, datos que guardan similitud con lo expuesto por Abumohor, pero difiriendo en que la artritis que desarrollaron los individuos aún es de tipo no especificada.

En estudio de Narváez (2009) denominado "Qué, cómo, cuándo y hasta cuándo tratar una artritis indiferenciada", en el que se menciona que artritis indiferencia o no identificada hace alusión a la artritis que según los criterios de clasificación del American College of Rheumatology aún no puede ser clasificada dentro de un diagnóstico específico, y que individuos que presentan este tipo de artritis pueden desarrollar a posterior una artritis reumatoidea $u$ otra enfermedad articular inflamatoria que se pueda identificar con mayor facilidad. Además, hace referencia a datos estadísticos de los pocos estudios que existen sobre cómo es la evolución de la artritis indiferenciada, en los cuales se reflejan que alrededor del 13 al $54 \%$ de individuos que manifiestan este tipo de artritis pueden ingresar a remisión de forma esporádica, pero que el $27 \%$ incluso hasta un $54 \%$ de ellos pueden evolucionar a artritis reumatoidea o a otras enfermedades articulares, mientras un 19 al 58\% se caracterizan por la persistencia de artritis indefinida sin que esta cambie. 
Gráfico 1. Manifestaciones articulares presentadas en individuos del grupo caso positivos a dengue.

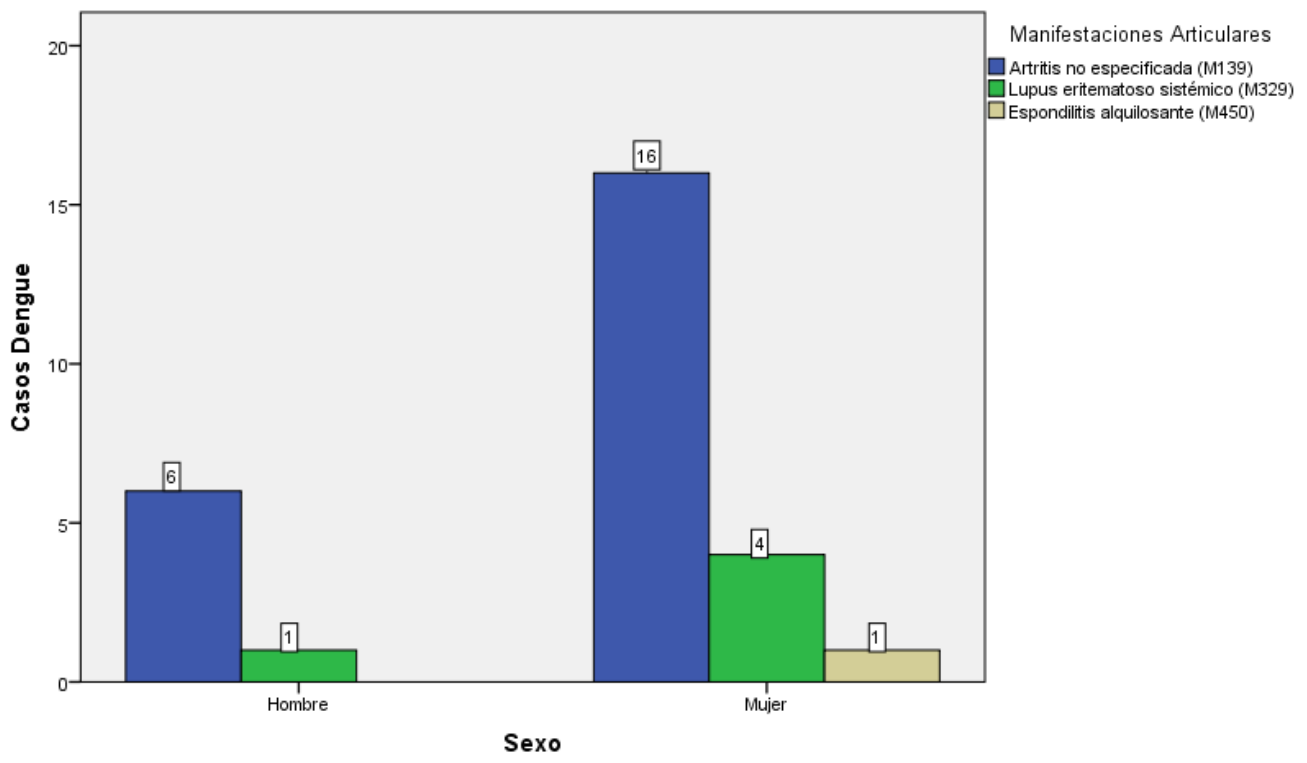

Fuente: Autores.

Gráfico 2. Manifestaciones articulares presentadas en individuos del grupo caso negativos a dengue.

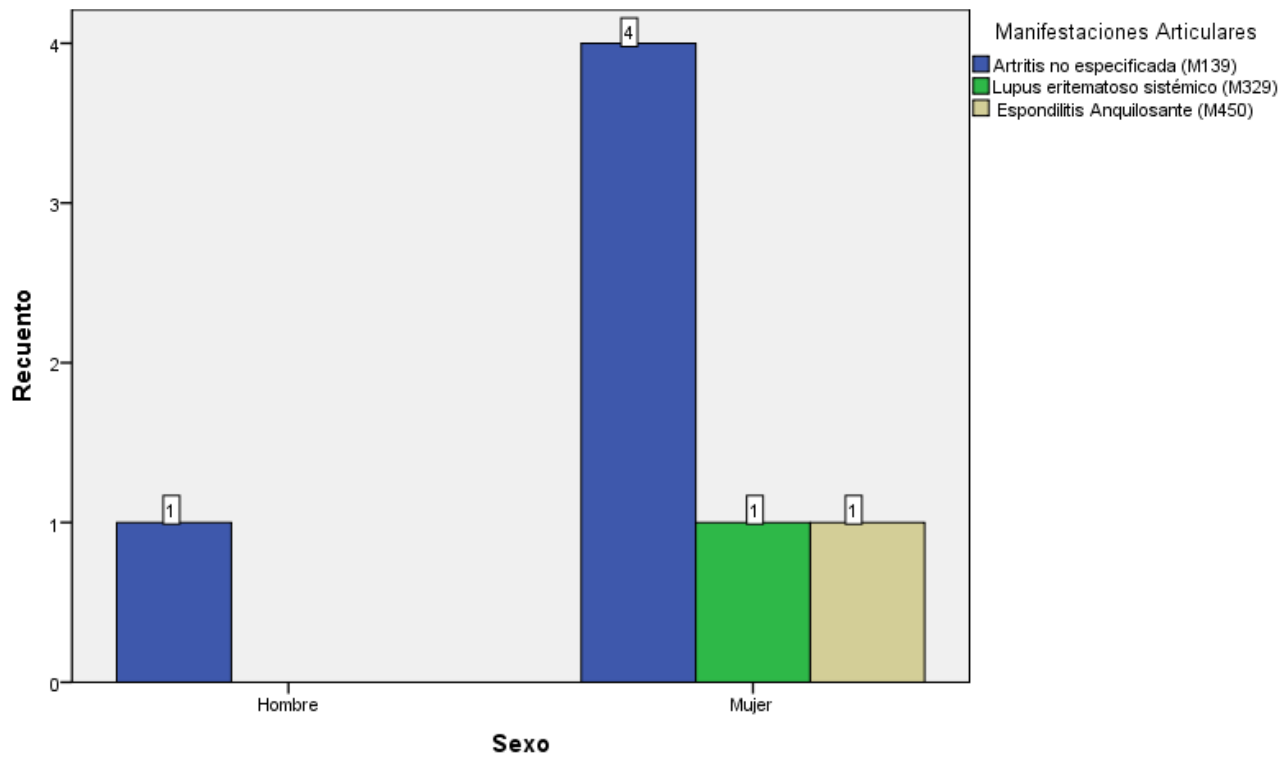

Fuente: Autores.

Al realizar una comparación entre los gráficos 1 y 2 se refleja una mayor incidencia de infección por dengue (28 casos) entre los individuos que desarrollaron manifestaciones articulares crónicas, además se muestra que la incidencia de este fue alta entre los individuos que desarrollaron artritis no especificada (22 individuos), basados en estos hallazgos es posible pensar que los individuos que desarrollaron artritis 
no especificada puedan desarrollar artritis reumatoidea o algún otro tipo de manifestación articular inflamatoria en algún momento de su vida como lo asevera Narváez en su estudio.

Así también, de acuerdo con Ruiz y Sanmartí (2012) en su estudio referente al Tabaco y otros factores ambientales en la artritis reumatoidea, se menciona que las características socioeconómicas de un individuo (Ingreso económicos, nivel de educación, actividad laboral etc.) están relacionadas en cómo se pueda desarrollar una manifestación articular como la artritis reumatoidea he incluso considera que estos serían un factor clave que aumenta el riesgo de desarrollar la misma. Por otra parte, menciona la relación existente entre consumo de tabaco y otros factores que puedan desencadenar la misma, para esto hace alusión a dos estudios prospectivos que datan de los años 90 en que se registraron datos de un estudio de cohortes de 121.700 enfermeras en que se evidenció que las que consumían tabaco eran ligeramente más propensas de desarrollar manifestaciones articulares como la artritis que las que no compartían este hábito, así también menciona los datos del segundo estudio en el que de 50.000 individuos investigados se identificó que los fumadores activos eran más propensos a desarrollar artritis reumatoidea seropositiva.

Bajo esta perspectiva, en lo que respecta a los factores sociodemográficos en nuestro estudio se evidenciaron datos registrados en la tabla 1 como ingresos económicos, educación, y consumo diario de tabaco, mismo que reflejan que el mayor tipo de ingresos percibidos por individuos que presentaron manifestaciones articulares fueron ingresos de tipo medio o incluso bajos, así también se estableció que el nivel de educación no superaba el bachillerato, y finalmente que en la mayoría de los individuos estudiados que presentaron manifestaciones articulares (21 de ellos) el consumo de tabaco era de entre 1 a 10 cigarrillos diarios; siendo estos factores junto a los ya mencionados del dengue puntos clave a considerar para el desarrollo de las manifestaciones articulares crónicas, y además si se considera que las condiciones de vida de los 
individuos se ven en gran medida determinados por la cantidad de ingresos percibidos estos pueden ser factores que determinen cual es la cantidad de recursos económicos que consideren adecuados destinar al cuidado de su salud, incluyendo el cuidado que se brinde a evitar el contagio de dengue, que como se plantea puede ser el detonante para que se desarrollen a posterior manifestaciones articulares.

Basados en los datos ya expuestos y en los hallados del cálculo de chicuadrado para las variables estudiadas y para dengue reflejados en la tabla 1, es posible establecer que hay una probabilidad mayor del 99\% de que haya una asociación entre el dengue y el desarrollo de manifestaciones articulares crónicas. Además, si consideramos resultados del OR se puede manifestar que el dengue es un determinante 11,44 veces mayor de desarrollar una manifestación articular crónica para un individuo que ha padecido al menos una infección de dengue en algún momento.

Con este resultado es oportuno poner en consideración continuar con esta investigación a futuro con el fin de buscar datos más precisos que puedan aseverar con mayor precisión que tan estrecha es la relación que guarda el dengue con el desarrollo de manifestaciones articulares crónicas, así como establecer cuál es la causa principal para que el dengue desarrolle dichas manifestaciones, para eso es preciso pensar tomar un grupo más grande de individuos a investigar que el que se tomó para este estudio, ya que así es más probable encontrar datos que permitan buscar una relación más estrecha entre el dengue y las manifestaciones articulares. Así también, se puede consideran el realizar exámenes clínicos que permitan buscar datos que den luz a las dudas planteadas, así como establecer cuál o cuáles son los serotipos involucrados en el desarrollo de las manifestaciones articulares. En el caso del presente estudio a los individuos control fue posible realizarles pruebas que confirmen la infección de dengue, pero, en lo concerniente a los individuos del grupo caso solo se contó con la información registrada en sus historias clínicas, además de las entrevistas realizadas a una parte de ellas, presentándose la imposibilidad de contactar en su 
totalidad a los mismos, siendo esto una posible fuente de sesgo, pues entre los casos el antecedente de dengue se recuperó de la historia clínica o vía entrevista.

Por otra parte, si consideramos lo mencionado por Abumohor (2012) en su estudio que muestra que los criterios creados en 1987 por el Colegio Americano de Reumatología que sirven para lograr diferenciar enfermedades de tejidos conectivos que cursan con artritis respecto de la artritis reumatoidea, mismos que fueron modificados y actualizados por la Liga Europea contra el Reumatismo y el American College of Rheumatology (EULAR/ACR) en el año 2010, y que permiten identificar de forma temprana el desarrollo de la mismas antes que comience a manifestarse la erosión (menos de 6 meses), es preciso considerar buscar una forma de establecer cuanto tiempo debe trascurrir desde el momento en que se presenta la infección del dengue hasta el desarrollo de la manifestación articular crónica, para así crear protocolos que permitan evitar el desarrollo de estas, o en tal caso buscar un tratamiento temprano que permita el control de las manifestaciones articulares, todo esto con la finalidad de mejorar las condiciones de vida de los pacientes.

Lamentablemente en el presente estudio no se pudo establecer una línea de tiempo entre la infección del dengue $y$ el momento en que comenzaron las manifestaciones articulares presentadas en el grupo caso, pues varios de los registros obtenidos no contaban con fechas exactas del momento en que se presentó la infección, y al consultar con los individuos si recordaban la fecha en la que se dio el evento estos mencionaban no recordarlo con exactitud.

\section{CONCLUSIONES}

1. Con el estudio realizado se evidenció que el dengue es un determinante que se debe considerar para la aparición de enfermedades articulares crónicas, pues con el cálculo del OR se demostró que este provoca un riesgo 11,44 veces superior de padecer algún tipo enfermedad articular crónica en pacientes que reportaron dengue en algún momento de su vida. 
2. Entre los grupos casos y control se evidenció que la mayoría de los individuos que conformaron los grupos de estudios ( $80 \%$ y $25,9 \%$ respectivamente) presentaron al menos 1 infección de dengue, volviendo al dengue un factor estadísticamente significativo (Chicuadrado de 32.89) de considerar para las manifestaciones articulares crónicas

3. En relación con las condiciones socioeconómicas de los individuos caso y control fue posible establecer que en ambos grupos el tipo de ingreso que más se percibe es de tipo medio, encontraste de los ingresos de tipo bajo y alto, siendo este último no percibido por ningún miembro del grupo caso y si por una parte del grupo control, volviendo al tipo de ingresos que puede percibir un individuo un factor significativo (Chi-cuadrado de 94.012) de considerar.

4. Respecto de antecedentes clínicos como el consumo de tabaco de los grupos caso control se estableció que mientras los individuos del grupo caso consumen entre 1 y 10 cigarrillos diariamente volviendo este hábito un factor importante y estadísticamente significativo (Chi-cuadrado de 51.00) de considerar para la predisposición de las manifestaciones articulares crónicas evidenciadas, los individuos del grupo control en su mayoría no consumen el mismo.

\section{BIBLIOGRAFÍA}

Abumohor, P. G. (2012). Enfermedades del tejido conectivo: Importancia del diagnóstico precoz. Revista Médica Clínica Las Condes, 23(4), 391-400. https://www.elsevier.es/esrevista-revista-medica-clinicalas-condes-202-pdfS0716864012703309

American College of Rheumatology. (2019). Espondiloartritis. https://www.rheumatology.org /I-Am-A/Patient-

Caregiver/Enfermedades-yCondiciones/Espondiloartritis

Bartolomé, S., Rodríguez, J., Bassy, N., \& Hornillos, M. (s. f.). Artritis Inflamatorias. Sociedad Española de Geriatría y Gerontología. Recuperado 10 de agosto de 2021, de https://www.segg.es/downloa d.asp?file=/tratadogeriatria/P DF/S35-05\%2068 III.pdf.

Biblioteca Nacional de Medicina de los EE. UU. (s. f.). Artritis idiopática juvenil. MedlinePlus. Recuperado 6 de octubre de 2021, de 
https://medlineplus.gov/spani sh/ency/article/000451.htm

Choque, F., Huamaní, F., \& Canelo, C. (2016). Síntomas crónicos tras episodio de dengue, una necesidad de investigación. Scielos.

https://www.scielosp.org/pdf/r pmesp/2016.v33n1/181-182

Colmán, N. (2017, 27 junio). Impacto de la infección por dengue en pacientes con enfermedades autoinmunes. Revisión de casos | Revista Paraguaya de Reumatología. Revista paraguaya de reumatología. http://www.revista.spr.org.py/i ndex.php/spr/article/view/69

Contreras, M., Rincón, M., Guillén, A., Moreira, R., \& Callejas, D. (2021). Aspectos genéticos del virus del dengue. QhaliKay. Revista de Ciencias de la Salud, 5(2), 79-88. https://revistas.utm.edu.ec/ind ex.php/QhaliKay/article/view/ 3496/3354

Cruz, Y., \& Montero, N. (2019). Calidad de vida en pacientes ecuatorianos con artritis reumatoide: un estudio transversal. Reumatología, 296.

Espinosa, R., Alcántar, J., Arce, C., Chávez, L., Esquivel, J., Gutiérrez, J., de la Lanza, L., Martínez, J., Méndez, C., Robles, M., Santillán, E., Torres, F., Sosa, J., Aldrete, J., \& Romero, A. (2018, mayo). Reunión multidisciplinaria de expertos para el diagnóstico y tratamiento de la osteoartritis. Actualización basada en evidencias. Medigraphic. https://www.medigraphic.com /pdfs/medintmex/mim2018/mim183m.pdf

Frantchez, V., Fornelli, R., Sartori, G., Arteta, Z., Cabrera, S., Sosa, L., \& Medina, J. (2016, abril). Dengue en adultos: diagnóstico, tratamiento y abordaje de situaciones especiales. SciELO. http://www.scielo.edu.uy/sciel o.php?script=sci_arttext\&pid= S1688-03902016000100006

Feced Olmos, C. M., Fernández Matilla, M., Robustillo Villarino, M., de la Morena Barrio, I., \& Alegre Sancho, J. J. (2016). Afectación articular secundaria a infección por virus de Epstein-Barr. Reumatología Clínica, 12(2), 100-102.

https://doi.org/10.1016/j.reum a.2015.05.014

Lozano, J. (2001). Artritis reumatoide (I). Etiopatogenia, sintomatología, diagnóstico y pronóstico. Offarm, 20(8), 94100.

https://www.elsevier.es/esrevista-offarm-4-articuloartritis-reumatoide-ietiopatogenia-sintomatologia13018371

Mandell, Douglas, \& Bennett. (2020). Enfermedades infecciosas. Principios y práctica (Novena 
ed.). $\quad$ El Sevier. https://clinicalkey.puce.elogim .com/\#!/content/book/3-s2.0B978849113499200103X?scr ollTo=\%23hl0001695

Masís, R., \& Jiménez, M. P. (2019). Abordaje del paciente con sospecha de patología reumática. Revista Clínica de la Escuela de Medicina UCRHSJD, $\quad$ 9(3), 1-5. https://www.medigraphic.com /pdfs/revcliescmed/ucr2019/ucr193a.pdf

Mateo, L., \& Roure, S. (2019). Artritis crónica en la infección por virus de Chikunguña. Reumatología Clínica, 15(2), 113-116.

https://doi.org/10.1016/j.reum a.2017.06.012

Mestanza. (2017). Artritis reumatoide. Scielo, 9.

Ministerio de Salud Pública del Ecuador. (s. f.-b). Ecuador en alerta para prevenir el contagio del dengue. Recuperado 28 de diciembre de 2020, de https://www.salud.gob.ec/estr ategia-nacional-de-controldel-dengue/

Narváez, F. (2009). Qué, cómo, cuándo y hasta cuándo tratar una artritis indiferenciada. Reumatología Clínica, 5, 3139.

https://doi.org/10.1016/j.reum a.2008.12.007

OMS. (2017). Dengue: guidelines for diagnosis, treatment, prevention and control. Geneva: World Health Organization.

Organización Mundial de la Salud. (2020, septiembre 15). Chikungunya. https://www.who.int/es/newsroom/factsheets/detail/chikungunya

Organización Mundial de la Salud. (2021, 19 mayo). Dengue y dengue grave. https://www.who.int/es/newsroom/factsheets/detail/dengue-andsevere-dengue

OPS. (1 de marzo de 2018). Number of reported cases of dengue and dengue hemorrhagic fever (DHF), Region of the Americas (by country and subregion) 1980-2018.

Obtenido de http://www.paho.org/data/inde x.php/en/mnutopics/indicadores-dengueen/dengue-nacional-en/252dengue-pais-ano-en.html

Romero, M., Stajnsznajder, P., Cassan, A., \& Torralba, A. (2010). Artritis reumatoide Información para pacientes y familiares [Libro electrónico]. Letramédica SCP. http://www.conartritis.org/wpcontent/uploads/2012/05/infor macion_actualizada_paciente s_familiares.pdf

Ruiz, V., \& Sanmartí, R. (2012). Tabaco y otros factores ambientales en la artritis 
reumatoide. Reumatología

Clínica, 8(6), 342-350.

https://doi.org/10.1016/j.reum

a.2012.02.011

Sociedad Española de Medicina

Interna. (s. f.). Lupus

eritematoso sistémico.

Recuperado 6 de octubre de

2021, de

https://www.fesemi.org/inform

acion-pacientes/conozca-

mejor-su-enfermedad/lupus-

eritematoso-sistemico-les

Velandia, M., \& Castellanos, J. (2011). Virus del dengue: estructura y ciclo viral. Scielo. http://www.scielo.org.co/pdf/in f/v15n1/v15n1a06.pdf

Suárez, A., \& Llorente, M. (2012). Diagnóstico y seguimiento de la artritis reumatoide. Seqce.es.

http://www.seqc.es/download/ tema/7/3320/3778230/59116 6/cms/tema-6-diagnostico-yseguimiento-de-la-artritisreumatoide.pdf/ 